\title{
Implant Dentistry Research Guide: Basic, Translational and Clinical Research
}

\author{
Ahmed Ballo \\ Publisher: Nova Science Publishers, Inc. \\ Language: English \\ ISSN: 978-1-61942-503-3 \\ Edition: 1/e \\ Publish Year: 2012 \\ Pages: 567, illustrated \\ Price: $\$ 215.00$
}

Dr. Ballo's book is the result of a collaboration involving international clinicians and scientists in the field of implant dentistry and guided-bone-regeneration research.

The Implant Dentistry Research Guide is a comprehensive book with a complete update on various methods and techniques used in this field.

The Guide has 6 part and 21 chapters.

Part I, chapter one presents relevant information from the fields of bone biology and osseointegration.

Part II, the next two chapters provide the general guidelines for topographical data and characterization of implant surfaces, and an overview of the approaches and methods to the surface structure, chemistry, and oxide thickness of the clinically available titanium implants.

The next three chapters which make up Part III describes procedures of using simulated body fluid to determine the bioactivity of a new implant candidate material; which cellculture models can be used to investigate the process of osseointegration and in vitro testing methods of implant prosthodontics evaluation.

Part IV and its five chapters reveal the research made on animal models. It tackles a tool to be concretely implemented in a self-reflection sheet before starting an animal trial; the strengths and limitations of the commonly small laboratory models frequently used and an overview of the different animal models used in alveolar bone and maxillary sinus augmentation research. Ethical issues raised

Florin Eugen by animal testing and the moral dilemma

Constantinescu offered by the 3 Rs: Reduction, Refinement, DDS,PhDStudent and Replacement end this part.

ROPOSTURO Methods for bone-implant interface analysis Holistic Dental Medical Institute E-mail:

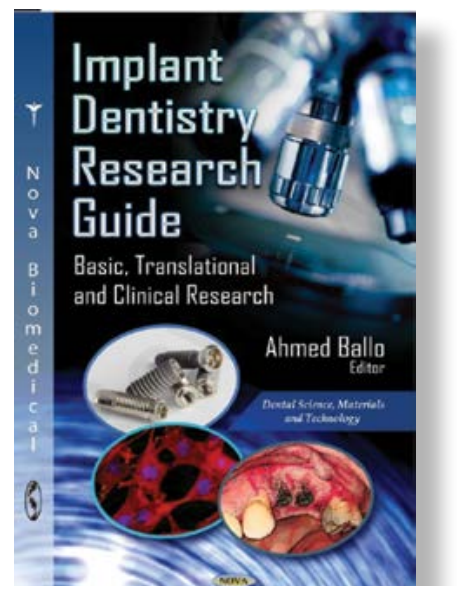

are presented in the next five chapters in Part $\mathrm{V}$.

It describes the theoretical principles and practical techniques used to analyze the bone-implant interface, the current application of micro-CT in different areas of the bone and the biomaterial research, the application of electron and ion beam microscopes for implant surface and boneimplant interface characterizations, the molecular biological techniques provide the tools necessary to further clarify the underlying mechanisms of osseointegration, and computer enginereering methods to evaluate the biomechanical behavior of the osseointegrated implants.

In Part VI, the last five chapters develop the human clinical research: the general principles of randomized controlled trials are discussed in detail, inclusion and exclusion criteria used, methods available to monitor the biological performance of a biomaterial, some relevant aspects of twodimensional radiography, and noninvasive assessment of implant stability for the clinical success of osseointegrated denial implants measurement by resonance frequency analysis (Osstell).

Finally, an appendix of standard abbreviations is also included.

Dr. Ballo with the support of the international dental community has published The Implant Dentistry Research Guide which is a successful attempt to promote the idea of improving patient care in the field of oral implantology and guided bone regeneration. 